\title{
Large brain effective network from EEG/MEG data and dMR information
}

\author{
Brahim Belaoucha \\ Université Côte d'Azur \\ Inria \\ France
}

\author{
Théodore Papadopoulo \\ Université Côte d'Azur \\ Inria \\ France
}

\begin{abstract}
Over the past 30 years, neuroimaging has become a predominant technique. One might envision that over the next years it will play a major role in disclosing the brain's functional interactions. In this work, we use information coming from diffusion magnetic resonance imaging (dMRI) to reconstruct effective brain network from two functional modalities: electroencephalography (EEG) and magnetoencephalography (MEG).
\end{abstract}

\section{INTRODUCTION}

MEG and EEG imaging techniques record the brain cortical activity in real time. Localizing active regions from these measurements, in the source distributed model, involves solving an ill-posed inverse problem. Prior on the source space must be set to obtain a unique solution.

Minimum norm estimate (MNE) is one of the most used inverse solvers because it leads to a linear inverse operator $[1,2]$. It results to widely extended active regions which are, biologically, not plausible. Among other solvers, we can find the minimum current estimate (MCE). It provides a focal reconstruction but is non-smooth in time because the sparse prior is applied at each time sample independently. Mixed-norm estimate (MxNE) [3] was introduced to overcome this limitation. It provides spatial focality, by applying $l_{1}$-norm on the source space, and temporal smoothness, by using $l_{2}$-norm on time courses.

Functional connectivity analysis has been traditionally implemented at the sensor level, but lately, a number of studies have begun to use source-space analysis. It has the potential of providing more accurate information regarding regions' functional in- teractions $[4,5,6]$. Some of the functional measures (e.g. Directed Transfer Function (DTF) and Partial Directed Coherence (PDC)) can be derived from multivariate autoregressive model (MAR) which is more than a combination of purely temporal and purely spatial correlation, as it also takes into account the time-lagged correlation between sources.

In [4], the authors constrain the dynamics of the sources by a MAR model. They assume that there exist only one time-lag, which depends on the length of anatomical connections, between a pair of regions. In [6], the authors use a variant of MxNE constrained with a MAR model of order 1 to reconstruct regions' interactions. In both works, complex time courses can not be reconstructed because of limits in the degree of freedom of the model. In this work, we generalize the work of [6] by constraining the dynamics of the cortical regions with higher MAR model whose matrix elements are constrained by the anatomical connectivity and neighbors. We call it iterative source and dynamics reconstruction of order $p$ (iSDR(p)). We test and compared our method with real and synthetic data.

\section{Method}

Under the quasi-static approximation of the Maxwell's equations, the EEG/MEG data $M_{t}$ is a linear superposition of the source signals $J_{t}$ at the same time $t$ :

$$
M_{t}=G J_{t}+\varepsilon_{t},
$$

where $G \in \mathbb{R}^{m \times n}$ ( $m$ : size of sensor array, $n$ : size of source space) is the lead-field matrix and 


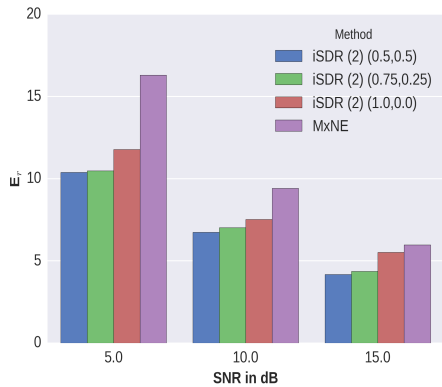

(a)

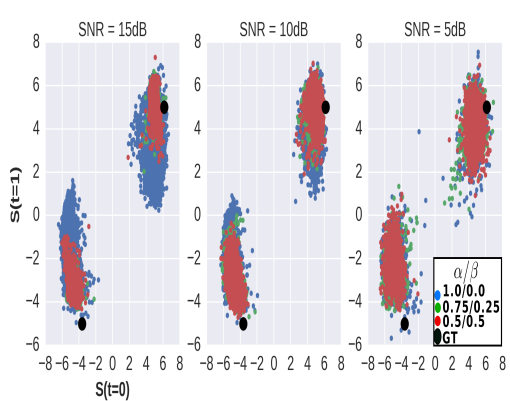

(b)

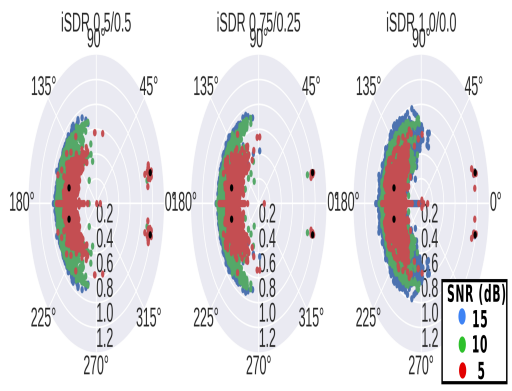

(c)

Fig. 1: In (a), mean, over 100 simulations, of reconstruction error $E_{r}$ of $\mathrm{MxNE}$ and iSDR(p=2) with different initialization of $A$ and SNR levels. (b) Estimated initial values of $S_{1}$ and $S_{2}$ using $\operatorname{iSDR}(p=2)$. (c) Eigenvalues of the companion matrix. The results are shown at 3 different SNR. Black dots represent ground truth values.

$\varepsilon_{t}$ is $\mathcal{N}\left(0, \Sigma_{c}\right)$. To reduce the problem complexity, we assume a constant activation per cortical region. This reduces the size of the lead-field to $m \times K$, where $K$ is the number of cortical regions. We use the mutual nearest neighbor parcellation algorithm presented in $[7,8]$ to divide the cortical surface into functional regions from dMRI. The source dynamics is constant during a time window $T$ and it follows MAR model of order $p$ :

$$
J_{t}=\sum_{i=1}^{p} A_{i} J_{t-i}+\omega_{t}
$$

where $\omega_{t}$ is $\mathcal{N}\left(0, \Sigma_{s}\right), p$ is a positive integer that defines the order of the MAR model, $A_{i}$ is a $K \times K$ matrix which relates sources' current values to its pasts. The only nonzero effective interactions in $A$ corresponds to either structurally connected or neighboring regions. The behavior of the MAR model is obtained from the companion matrix $\boldsymbol{\Phi}$ :

$$
\boldsymbol{\Phi}=\left[\begin{array}{ccccc}
A_{1} & A_{2} & \cdots & A_{p-1} & A_{p} \\
I & & & & \\
& \ddots & & &
\end{array}\right]
$$

where $I$ is the $K \times K$ identity matrix. The MAR model is stable (stationary) if all eigenvalues of $\boldsymbol{\Phi}$ are inside the unit circle and nonstationary if it has eigenvalues on the unit circle. The model is unstable if at least one eigenvalue is outside the unit circle. The sources estimates $J$ are obtained by minimizing the following functional given a MAR model:

$$
U(J)=\left\|M_{v}-\mathbf{G}_{\mathbf{d}} J_{v}\right\|_{2}^{2}+\lambda\|J\|_{21}
$$

where $M_{v}=\left[M_{p+1}, \cdots, M_{T}\right], J_{v}=\operatorname{vec}(J)=$ $\operatorname{vec}\left(\left[J_{1}, \cdots, J_{T-1}\right]\right)$ and $\|J\|_{21}=\sum_{i} \sqrt{\sum_{j} J(i, j)^{2}}$ i.e. $l_{2}$-norm over time and $l_{1}$-norm over space.

$$
\mathbf{G}_{\mathbf{d}}=\left[\begin{array}{cccccc}
G_{1} & G_{2} & \cdots & G_{p} & & \\
& \ddots & \ddots & \ddots & \ddots & \\
& & G_{1} & \cdots & G_{p-1} & G_{p}
\end{array}\right]
$$

where $G_{i}=G A_{i}$. The MAR elements are obtained by fitting the source estimates to the MAR model:

$$
\underset{A_{1}, \cdots, A_{p}}{\operatorname{argmin}} \sum_{t=p+1}^{T}\left\|J_{t}-\sum_{i=1}^{p} A_{i} J_{t-i}\right\|_{2}^{2}
$$

We solve iteratively $\mathrm{Eq} 4$ and 6 until $\left\|J^{k}-J^{k-1}\right\|<\epsilon$ where $J^{k}$ is the reconstructed sources at iteration $k$.

\section{DATA}

MEG and EEG were measured using 306 system (102 magnetometers, 204 planar gradiometers), and 70 EEG channels recorded EEG data simultaneously. Stimuli were presented in six 7.5 min runs. The face stimuli contain two sets of 300 gray scale photographs, half from unfamiliar people (unknown to the participants) and the remaining from famous people. In a third condition, 150 photographs of scrambled faces are obtained from either famous or unfamiliar people. The reader is referred to [9] for more details.

In this work, we are interested in localizing face recognition areas. For this reason, we use only the measurement acquired when using photos of famous people subtracted to the ones obtained when 
using scrambled faces. Low pass filter of cut-off frequency $35 \mathrm{~Hz}$ was used to smooth the data. The MEG/EEG forward problem, lead field matrix $G$, is obtained using OpenMEEG [10, 11].

\section{RESULTS AND DISCUSSION}

\section{A. Simulation}

We use a random lead-field matrix of size $20 \times 500$ and whose elements are obtained from a normal distribution $\mathcal{N}(0,1)$. Each region is connected randomly to 4 different regions. We activate 50 random pairs of regions, one pair at a time, 100 times at different noise levels (signal-to-noise ratio $(\mathrm{SNR})=\{15,10,5\} \mathrm{dB})$ using the following simple MAR model: $\left[\begin{array}{c}S_{1} \\ S_{2}\end{array}\right]_{t}=$ $\left[\begin{array}{cc}0.43 & 0.24 \\ -0.24 & 0.42\end{array}\right]\left[\begin{array}{l}S_{1} \\ S_{2}\end{array}\right]_{t-1}+\left[\begin{array}{cc}0.47 & 0.12 \\ -0.12 & 0.46\end{array}\right]\left[\begin{array}{c}S_{1} \\ S_{2}\end{array}\right]_{t-2}$, with $J_{0}\left(S_{1}\right)=6.15, J_{0}\left(S_{2}\right)=-3.64, J_{1}\left(S_{1}\right)=$ $-J_{1}\left(S_{2}\right)=5 n A$ i.e. $J_{t}=A_{1} J_{t-1}+A_{2} J_{t-2}$. We estimate the sources and their interactions by initializing $A_{2}$ and $A_{1}$ with different values to have an insight about the effect of the MAR's initialization on the resulting estimate. We use the following combinations: $A_{2}=\beta I$ and $A_{1}=\alpha I$ with $(\alpha, \beta) \in\left\{(1,0),\left(\frac{3}{4}, \frac{1}{4}\right),\left(\frac{1}{2}, \frac{1}{2}\right)\right\}$. The results are presented in Fig (1). Fig (1a) represents the reconstruction error computed as $\left\|J_{g t}-J_{r}\right\|_{2}$, where $J_{g t}$ and $J_{r}$ are the simulated ground truth and reconstructed activation, using $\mathrm{MxNE}$ and $\operatorname{iSDR}(\mathrm{p}=2)$ respectively. The estimated initial values of $S_{1}$ and $S_{2}$ obtained from iSDR(p=2) are shown in Fig (1b). Fig (1c) represents the nonzero eigenvalues of the estimated companion matrix $\boldsymbol{\Phi}$.

iSDR(p) outperforms MxNE especially in low SNR. For $(\alpha, \beta)=(1,0)$, iSDR favors the initial values $\left(J_{t=0}\right)$ to be close to 0 , see blue dots in Fig (1b), due to initializing $A_{2}=0$ i.e no interactions between $t$ and $t-2$. Setting $\beta$ to nonzero improves both the temporal reconstruction of signals and their dynamics (eigenvalues of $\boldsymbol{\Phi}$ ). iSDR could reconstruct accurately two eigenvalues, right side of the plane in Fig (1c). The remaining two are affected by the choice of $(\alpha, \beta)$. The results are less dispersed when $(\alpha, \beta)=\left(\frac{1}{2}, \frac{1}{2}\right)$ (see left side of Fig (1c)).
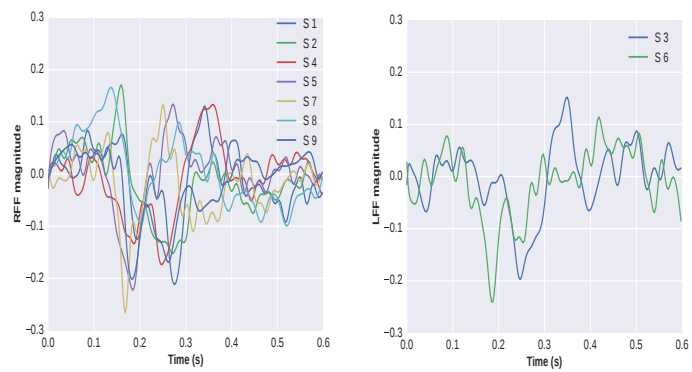

Fig. 2: Reconstructed activation in the right fusiform gyrus (RFF) of 7 subjects, left panel, and left FF (RFF), of 2 subjects using $\operatorname{iSDR}(p=2)$. The temporal courses are normalized with their $l_{2}$-norm for subjects comparison.

\section{B. Real data}

$\operatorname{iSDR}(\mathrm{p}=2)$ is applied to MEG data of 9 subjects from [9]. In [12], the authors show that subjects may have right or bilateral fusiform gyrus (FF) activation if they are right or left handed, respectively. In Fig 2, we show the estimated temporal course of the right/left FF (R/LFF). In our work, seven subjects show higher activation in the RFF, Fig (2) left, while only two show higher activation in LFF, Fig (2) right. Due to page limitation, we show only the effective connectivity of Subject 1, Fig (3).

Several works $[9,4]$ show that activation in the FF between 170 and $200 \mathrm{~ms}$ is observed when performing face recognition task which coincides with what we observe in this work. In the majority of subjects, regions in the superior posterior temporal lobe, medial parietal, temporal pole (TP), orbitofrontal (OF) are found to be active. This coincides with the findings of other works with the same dataset $[9,4]$. We have found interactions between TP and frontal lobe (FL), left and right FL, inferior occipital and temporal gyrus with OF.

We applied our method only with MAR model of order 2. Our approach provides functionally plausible results when tested with MEG data provided by [9].

\section{Conclusion}

In this work, we proposed a source reconstruction method constrained by multivariate autoregressive model from functional imaging EEG/MEG data. 


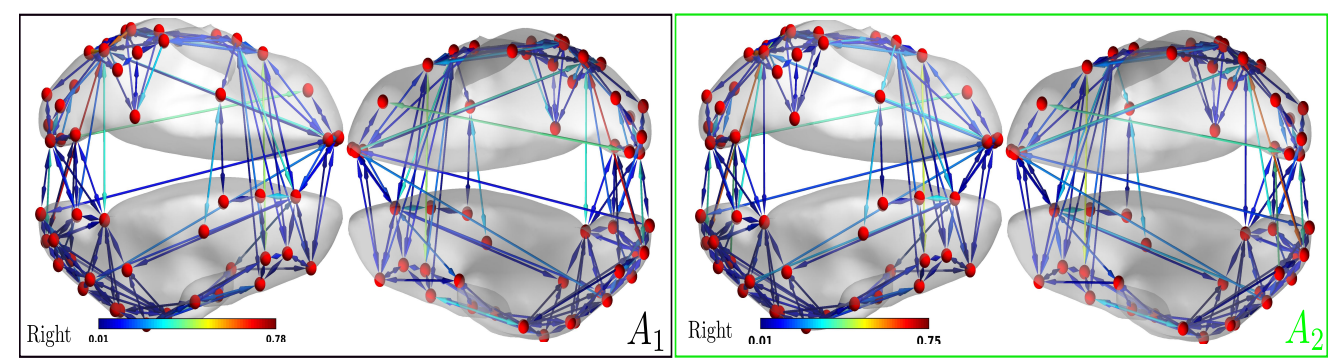

Fig. 3: Effective network of $A_{1}$, left, and $A_{2}$, right, obtained from iSDR(p=2) and MEG data [9] during face recognition task. Red spheres represent center of active cortical regions. Edges represent uni/bidirectional interactions. Self-interactions are not shown for visualization clarity. Edge's color represents its strength.

Application to real data of other mental tasks must be investigated and compared to physiological literature to validate our method. Information criterion can be used to obtain the order of MAR model.

\section{ACKNOWLEDGMENT}

This work has received funding from ERC under the European Union's Horizon 2020 research and innovation program (ERC Advanced Grant agreement No 694665 : CoBCoM) and French ANR contract Vibrations. We thank Dr. Daniel Wakeman and Prof. Richard Henson for providing the data.

\section{REFERENCES}

[1] J. Z. Wang et al. "Magnetic source images determined by a lead-field analysis: the unique minimum-norm least-squares estimation". In: IEEE Trans. Biomed. Eng 39.7 (1992), pp. 665-675.

[2] M. S. Hämäläinen et al. "Interpreting magnetic fields of the brain: minimum norm estimates". In: Med Biol Eng Comput 32.1 (1994), pp. 35-42.

[3] A. Gramfort et al. "Mixed-norm estimates for the M/EEG inverse problem using accelerated gradient methods". In: Phys. Med. Biol. 57.7 (Mar. 2012), pp. 1937-1961.

[4] M. Fukushima et al. "MEG source reconstruction based on identification of directed source interactions on whole-brain anatomical networks". In: NeuroImage 105 (2015), pp. $408-427$.
[5] K. Sekihara et al. "Source-Space Connectivity Analysis Using Imaginary Coherence". In: Electromagnetic Brain Imaging: A Bayesian Perspective. Cham: Springer International Publishing, 2015, pp. 139-169.

[6] B. Belaoucha et al. "Multivariate autoregressive model constrained by anatomical connectivity to reconstruct focal sources". In: Conf Proc IEEE Eng Med Biol Soc. 2016, pp. 4067-4070.

[7] P. Bellec et al. "Identification of large-scale networks in the brain using fMRI". In: $\mathrm{Neu}$ roImage 29.4 (2006), 12311243.

[8] B. Belaoucha et al. "Cortical Surface Parcellation via dMRI using Mutual Nearest Neighbor Condition". In: Proc IEEE Int Symp Biomed Imaging. Prague, Czech Republic, Apr. 2016, pp. 903-906.

[9] D. G. Wakeman et al. "A multi-subject, multi-modal human neuroimaging dataset". In: Scientific Data (2015).

[10] J. Kybic et al. "A common formalism for the Integral formulations of the forward EEG problem". In: IEEE Trans. Biomed. Eng 24.1 (Jan. 2005), pp. 12-28.

[11] A. Gramfort et al. "OpenMEEG: opensource software for quasistatic bioelectromagnetics". In: BioMedical Engineering OnLine 9.1 (2010), pp. 1-20.

[12] H. Bukowski et al. "Cerebral lateralization of face-sensitive areas in left-handers: Only the FFA does not get it right". In: Cortex 49.9 (2013), pp. $2583-2589$. 